\title{
Oligometastatic Malignant Solid Neoplasm
}

National Cancer Institute

\section{Source}

National Cancer Institute. Oligometastatic Malignant Solid Neoplasm. NCI Thesaurus.

Code C161019.

A malignant solid neoplasm that has metastasized to a limited number of sites- existing in a state intermediate between localized disease and widespread metastatic disease. 